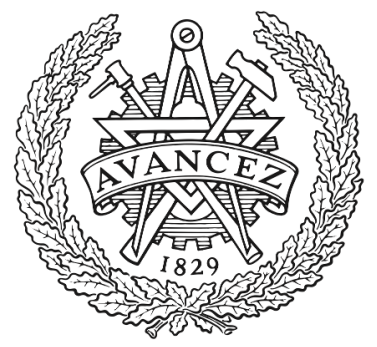

CHALMERS

UNIVERSITY OF TECHNOLOGY

\title{
Acoustic monitoring of rail faults in the German railway network
}

Downloaded from: https://research.chalmers.se, 2023-04-26 13:00 UTC

Citation for the original published paper (version of record):

Pieringer, A., Stangl, M., Rothhämel, J. et al (2021). Acoustic monitoring of rail faults in the German railway network. Notes on Numerical Fluid Mechanics and Multidisciplinary Design: 242-250 . http://dx.doi.org/10.1007/978-3-030-70289-2_24

N.B. When citing this work, cite the original published paper. 


\title{
Acoustic monitoring of rail faults in the German railway network
}

\author{
Astrid Pieringer ${ }^{1,2}$, Matthias Stangl ${ }^{1}$, Jörg Rothhämel ${ }^{1}$ and Thorsten Tielkes ${ }^{1}$ \\ ${ }^{1}$ DB Systemtechnik GmbH, Department of Acoustics, Vibrations, Aerodynamics and HVAC \\ (TT.TVE 34), Völckerstraße 5, Munich, Germany \\ ${ }^{2}$ Chalmers University of Technology, Applied Acoustics, 41296 Gothenburg, Sweden \\ astrid.pieringer@chalmers.se
}

\begin{abstract}
The early detection of rail surface defects such as squats, poor welds, or wheel burns is important to prevent further rail deterioration. In this paper, a methodology for acoustic monitoring of squats in the German railway network is proposed based on the measurement of axle box acceleration (ABA) on the DB noise measurement car (SMW) and the previously developed numerical model WERAN for wheel/rail interaction. Specific characteristics of squats in the ABA signals are determined with the model and verified by pass-by measurements combined with direct geometry measurements of the squats. Based on these results, a logistic regression classifier is devised for the detection of squats in the measured ABA signals of the SMW. Trained with simulated and measured data, the classifier identifies all of the known severe squats and $87 \%$ of the known light squats in the measured test data.
\end{abstract}

Keywords: Acoustic monitoring, Squats, Axle box acceleration, Wheel/rail interaction, Time-domain modelling, Noise measurement car, Machine learning.

\section{Introduction}

The early detection of rail surface defects such as squats, poor welds, or wheel burns is important to prevent further rail deterioration caused by large impact forces. Surface defects can be detected and measured optically by dedicated measurement trains, by ultrasound measurements or by visual inspection. These procedures give information about the geometry of the defect but cannot directly assess the severity in terms of the generated contact forces. Furthermore, early-stage defects are difficult to detect by these methods. An alternative to these direct measurements is the analysis of acoustic signals registered by monitoring systems on rolling stock. When the wheel rolls over a surface defect of the rail, the surface discontinuity leads to a relative motion of wheel and rail, causing vibrations and noise [1,2]. The discrete nature of the event and the generated large amplitudes distinguish the phenomenon from rolling noise and provide opportunities for the detection of the surface defect in vibration or noise signals. Both axle box acceleration (ABA) and under-coach noise measurements can be used for this purpose. An advantage of such monitoring systems is that the measured quantity is 
directly related to the wheel/rail contact forces indicating the severity of the rail faults. A prerequisite for detection is, however, that the surface defect passes through the wheel/rail contact area. Defects located outside the running band cannot be captured.

Only a few publications deal with the detection of rail faults by acoustic monitoring on rolling stock. These include the detection of squats [3] and rail welds [4] in ABA signals and the detection of squats by under-coach noise measurements [5]. The most promising approaches apply detailed time-domain models of the rolling contact to determine the specific characteristics of the faults in the ABA signals and exploit the simulation results to devise a detection procedure. In the case of squats in the Dutch railway network, high hit rates could be achieved with this strategy even for light squats [3].

A similar methodology is proposed in this paper for the detection of rail faults in the German railway network with the main focus on squats. The methodology is based on ABA measurements on the noise measurement car (SMW) [6] of DB Systemtechnik presented in Section 2 and the numerical model WERAN for wheel/rail interaction and noise $[7,8]$ described in Section 3. After adaption to the SMW, WERAN is applied to calculate the ABA signals due to squats of different severity and for different track parameters to find characteristic signatures in the time domain or in the time-frequency domain allowing to identify the fault (Section 4). In addition, a measurement campaign on a particularly bad track with several squats has been carried out providing measured signatures of squats in combination with direct geometry measurements of the faults (Section 5). Based on the simulated and measured results, a logistic regression classifier is devised to detect squats in the measured ABA signals of the SMW (Section 6).

\section{Noise measurement car "Schallmesswagen"}

The noise measurement car SMW (Fig. 1a) is employed to guarantee low rail roughness and hence a reduction in rolling noise on track sections called "specially monitored track" [6]. For this purpose, the SMW measures its own rolling noise. When the rolling noise reaches the intervention limit, the rails are acoustically ground. The rolling noise is measured with a microphone mounted above a hole in a semi-anechoic chamber directly above the measurement bogie (Fig. 1b). The wheels of the measurement bogie are unbraked and specially maintained to ensure low wheel roughness and thereby eliminate the influence of the wheel roughness on the rolling noise. Recently, the wheelsets of the measurement bogie have been equipped with accelerometers on the axle boxes (Fig. 1c). Vertical ABA is measured on all four axle boxes (and lateral ABA on two of them) with a sampling rate of $20 \mathrm{kHz}$. As the SWM travels about 50,000 km a year on the German railway network, it provides a large database of recordings.

(a)
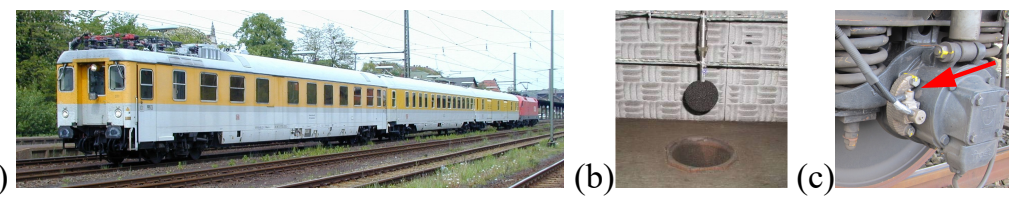

Fig. 1. Noise measurement car of DB Systemtechnik (a) equipped with a microphone above the measurement bogie (b) and accelerometers on the axle boxes of the measurement bogie (c). 


\section{The model WERAN}

WERAN (WhEel/RAil Noise) is a non-linear time-domain model for the calculation of wheel/rail interaction and noise [7,8], which includes a detailed 3D submodel of the wheel/rail contact based on Kalker's variational method [9]. Nevertheless, it is computationally efficient and allows for large parameter studies. This is achieved by employing a Green's function approach. Vehicle and track are represented by Green's functions that are pre-calculated from the receptances at the contact point by inverse Fourier transform. Instead of performing a time integration, the wheel and rail displacements are obtained by convoluting the time series of contact forces with the Green's functions. The Green's function of the track is a moving Green's function that includes the motion of the contact point on the rail [7]. The ABA signal is determined in a post-processing step by convoluting the contact forces with the Green's function calculated from the transfer receptance between the contact point on the wheel and the axle box.

Due to the Green's function approach, WERAN is easily coupled to different vehicle and track models as long as they are linear and time-invariant. In this study, the track model is the finite element (FE) model accounting for discrete supports presented in [10]. The model comprises a 60E2 rail represented by Rayleigh-Timoshenko beam elements. The discrete supports consist of railpads and sleepers on ballast. The vehicle model includes a complete wheelset of the SMW comprising the axle, two wheels of type BA093 and two brake disks, see Fig. 2. The primary suspension is also included, while all vehicle components above the primary suspension are represented as a static preload. The axle boxes are not modelled. Instead, it is assumed that the acceleration at a point on the axle $8 \mathrm{~cm}$ from the axle end approximately corresponds to the axle box acceleration. The wheel radius is $469 \mathrm{~mm}$ corresponding to a reprofiled wheel (while a new wheel has a radius of $475 \mathrm{~mm}$ ). The wheelset is modelled by 3165 axisymmetric finite elements using a commercial FE software. With this undamped FE model, the eigenfrequencies and eigenmodes have been calculated up to $10 \mathrm{kHz}$. Since a measurement of the transfer function from contact to axle box was not available, the modal damping has been determined from the measured radial and axial wheel receptances at the contact [11]. Modes that could not be identified in the measurements (involving in particular modes with axle bending which have higher damping) were assigned the approximate damping values proposed by Thompson [1]. The measured receptances were also used to update the eigenfrequencies while the eigenmodes were not changed. From this modal basis, the Green's functions of the wheelset were calculated.

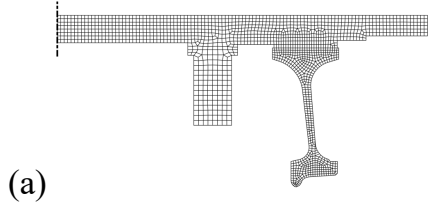

(a)

(b)

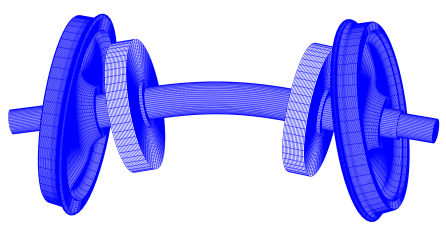

Fig. 2. Flexible wheelset model of the SMW: half of the 2D mesh of axisymmetric elements (a) and $3 \mathrm{D}$ visualization of the eigenmode at $244 \mathrm{~Hz}(\mathrm{~b})$. 
WERAN can consider both vertical and lateral wheel/rail interaction. In this study, only vertical wheel/rail interaction is included since this is considered sufficient for the excitation by rail surface defects.

\section{$4 \quad$ Simulated signatures of squats}

To determine the specific characteristics of squats in the ABA signals, a parameter study has been carried out with WERAN. The rail model has been adapted to the average measured track receptance from left and right rail at the shunting yard Munich North, see Section 5. This led to a rail pad stiffness $k_{\mathrm{p}}=400 \mathrm{MN} / \mathrm{m}$, rail pad damping $60 \mathrm{kNs} / \mathrm{m}$, ballast stiffness $k_{b}=200 \mathrm{MN} / \mathrm{m}$ and ballast damping $c_{b}=100 \mathrm{kNs} / \mathrm{m}$. The sleeper distance was $0.6 \mathrm{~m}$. The center of the squats was placed at a quarter of the sleeper bay at the top of the rail. In all simulations, the train speed was $80 \mathrm{~km} / \mathrm{h}$.

\subsection{Squat modelling}

The squats were modelled according to the following formula presented in [12]:

$z_{\mathrm{s}}(x, y)=-\frac{h}{2}\left(1+\cos \left(\pi \sqrt{\left(\frac{x}{a}\right)^{2}+\left(\frac{y}{b}\right)^{2}}\right)\right), x, y \in\left\{x, y: \sqrt{\left(\frac{x}{a}\right)^{2}+\left(\frac{y}{b}\right)^{2}} \leq 1\right\}(1)$,

where $h$ is the squat depth, and $a$ and $b$ are the half-length and half-width, respectively. An example of a 3D squat shape is given in Fig. 3, along with different longitudinal profiles for the same squat depth.
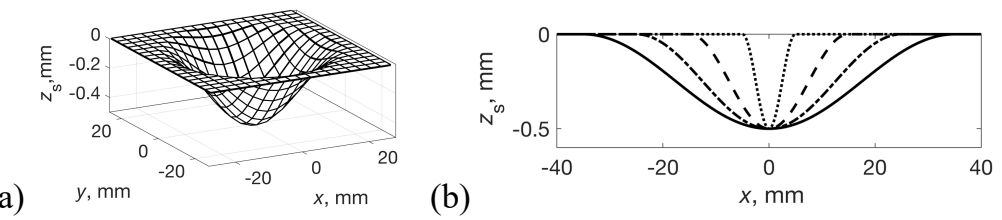

Fig. 3. Examples of squats according to Eq. (1): 3D shape for $h=0.5 \mathrm{~mm}$ and $a=b=25 \mathrm{~mm}$ (a) and longitudinal profile at $\mathrm{y}=0$ for $h=0.5 \mathrm{~mm}$ and $a=5,15,25$ or $35 \mathrm{~mm}(b)$.

\subsection{Influence of the squat dimensions}

ABA signals for rolling over circular squats (i.e. $a=b$ ) with depths ranging from 0.1 $1.4 \mathrm{~mm}$ and diameters ranging from $10-70 \mathrm{~mm}$ have been calculated. Except for the shallowest squats, the squat diameter has a larger influence on the maximum $\mathrm{ABA}$ and the shape of the ABA time signal than the squat depth. The influence of the squat diameter for a squat of depth $0.5 \mathrm{~mm}$ on the ABA time series is presented in Fig. 4a, where the center of the squat is located at position $0 \mathrm{~m}$. When the wheel enters the squat zone, unloading occurs in the contact force, leading to a decrease in acceleration. The subsequent impact entails a strong peak in the acceleration. The larger the squat diameter, the larger the peak magnitude and the width of the peak. The corresponding power 
spectra in Fig. 5a reveal that the dominating frequencies in the signals are in the main independent of the squat diameter. Leaving aside the smallest squat whose length corresponds to the length of the contact area, the ABA levels are also similar at high frequencies above $700 \mathrm{~Hz}$. Below larger differences occur.

(a)
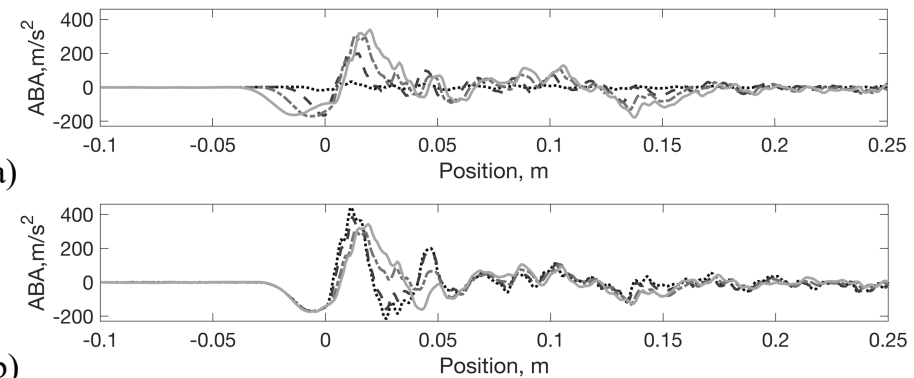

(b)

Fig. 4. Time series of ABA for rolling over a squat of depth $0.5 \mathrm{~mm}$ for different squat diameters: .... $10 \mathrm{~mm},---.30 \mathrm{~mm},---50 \mathrm{~mm},-70 \mathrm{~mm}$ (a); and for different rail pad stiffnesses: .... $55 \mathrm{MN} / \mathrm{m},-.-135 \mathrm{MN} / \mathrm{m},---400 \mathrm{MN} / \mathrm{m},-800 \mathrm{MN} / \mathrm{m}$, with squat diameter $50 \mathrm{~mm}$ (b).

(a)

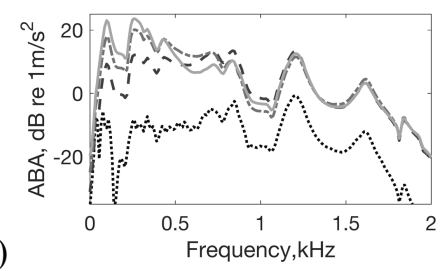

(b)

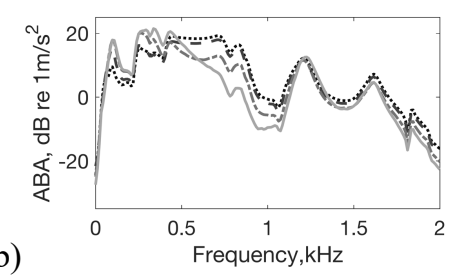

Fig. 5. Power spectrum of ABA for rolling over a squat of depth $0.5 \mathrm{~mm}$ for different squat diameters: .... $10 \mathrm{~mm},-.-30 \mathrm{~mm},---50 \mathrm{~mm},-70 \mathrm{~mm}$ (a); and for different rail pad stiffnesses: .... $55 \mathrm{MN} / \mathrm{m}$, -.- $135 \mathrm{MN} / \mathrm{m},---400 \mathrm{MN} / \mathrm{m},-800 \mathrm{MN} / \mathrm{m}$, with squat diameter $50 \mathrm{~mm}$ (b).

\subsection{Influence of the railpad stiffness}

The influence of the rail pad stiffness ranging from very soft pads $(55 \mathrm{MN} / \mathrm{m})$ to very stiff pads $(800 \mathrm{MN} / \mathrm{m})$ is shown in Figs. $4 \mathrm{~b}$ and $5 \mathrm{~b}$ for a squat of depth $0.5 \mathrm{~mm}$ and diameter $50 \mathrm{~mm}$. The softer the pads, the higher and narrower the first peak in the ABA time series. In the spectrum of the contact force (not shown here) a change of the rail pad stiffness leads to a shift in frequency of some of the peaks. Due to the resonant behavior of the wheelset, these frequency shifts are not prominent in the spectrum of the ABA signal. The main response is still at the same wheel modes. The level of the $\mathrm{ABA}$ spectrum is influenced by the rail pad stiffness mainly below $1 \mathrm{kHz}$.

\section{Measured acoustic signatures of squats}

In order to provide measured signatures of squats and data for validation of the model WERAN, a measurement campaign was carried out at the $5 \mathrm{~km}$-long northern passing 
track at the shunting yard Munich-North in March 2019. The detailed geometry of three severe and four light squats was measured with the rail roughness measurement device RM1200E proving $1.2 \mathrm{~m}$-long roughness lines with a longitudinal resolution of $0.5 \mathrm{~mm}$. Up to 11 parallel roughness lines with a distance of $3 \mathrm{~mm}$ have been measured for each squat. A severe squat (squat A) and a light squat (squat B) that were located on the right rail are presented in Fig. 6, together with roughness lines through the squat centers.

(a)

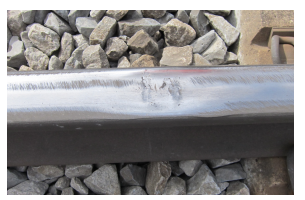

(b)

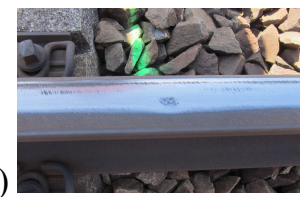

(c)

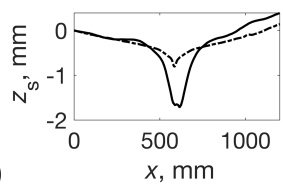

Fig. 6. Photograph of severe squat A (a) and light squat B (b) at shunting yard Munich North. Corresponding squat profiles at midline (c): - squat A, -.-. squat B.

In addition, the vertical track receptance was measured on the right and left rail at a location $40 \mathrm{~m}$ from squat $\mathrm{A}$ and $1.3 \mathrm{~km}$ from squat $\mathrm{B}$. It differed considerably between the two sides of the rail (not shown here) and is expected to vary along the track. Eight days later, pass-by measurements were carried out with the SMW. For four pass-bys in the main travelling direction and four in the opposite direction at $80 \mathrm{~km} / \mathrm{h}, \mathrm{ABA}$ signals were recorded on the SMW. At the time of the pass-by measurements, the rail at squat A had been equipped with an emergency fishplate connector for safety reasons.

(a)
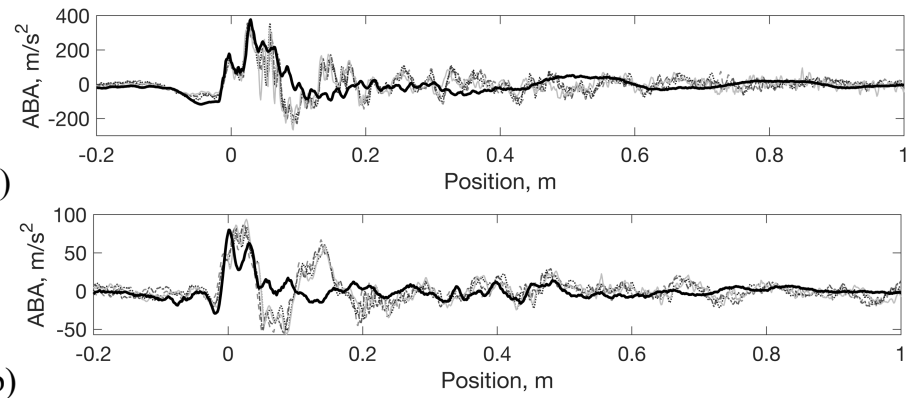

Fig. 7. Time series of ABA for rolling over squat $\mathrm{A}(a)$ and $\mathrm{B}(b)$ : four measured pass-bys in the main travelling direction at $80 \mathrm{~km} / \mathrm{h}$ (grey, different line styles) and simulation (black line).

As seen in Fig. 7, the measured ABA signals show good repeatability between passbys. Based on the measured squat geometries of squat A and B, WERAN has been used to predict the ABA signal. Here, the rail model was adapted to the track receptance measurement at the right rail. The comparison of simulated and measured signals is presented in Fig. 7 in terms of the ABA time signal and in Fig. 8 in terms of the spectrogram. The model predicts well the magnitude and width of the first ABA peak, but the simulated response is too highly damped. The measured and simulated spectrograms show similar patterns for the positions from $-0.6 \mathrm{~m}$ to $+0.6 \mathrm{~m}$ around the squat where roughness/geometry was measured. Some of the peaks appear, however, shifted 
in frequency, e.g. the measured peak at $1.4 \mathrm{kHz}$ appears at $1.2 \mathrm{kHz}$ in the simulated response. These differences are mainly attributed to the uncertainty about eigenfrequencies and damping of the wheelset modes involving bending of the axle, which could not be measured, see Section 3. Additionally, the rail receptance at the squat may have differed from the one at the measurement position, and the emergency fishplate connector at squat $\mathrm{A}$ has not been modelled.
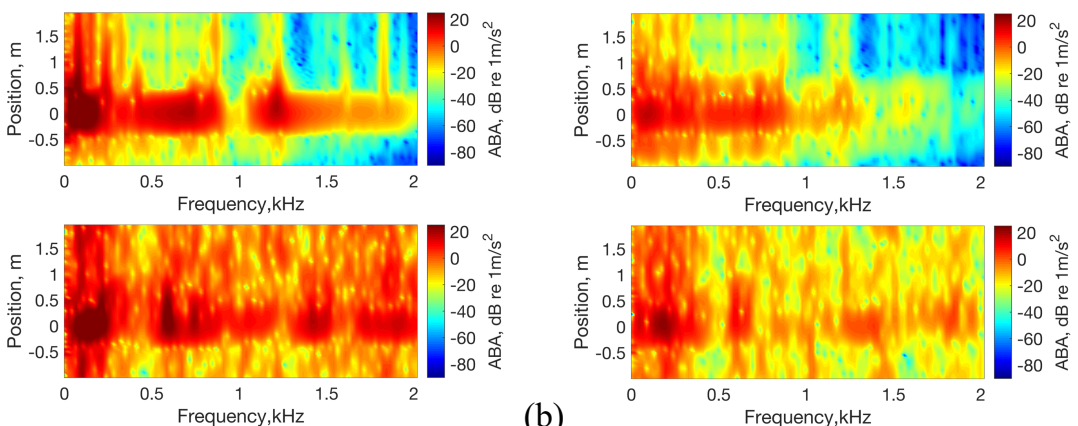

(a)

(b)

Fig. 8. Spectrograms of ABA for rolling over squat A $(a)$ and B $(b)$ : simulation (upper row) and one selected measured pass-by in the main travelling direction (lower row) at $80 \mathrm{~km} / \mathrm{h}$.

\section{Detection algorithm}

As seen in Sections 4 and 5, the impact at the squat leads to a temporary increase in the ABA signal over a wide frequency range. For the same wheelset, the main excited frequencies are relatively independent of the squat dimensions and the rail support properties. Against this background, a pattern recognition algorithm based on machine learning seems a promising strategy to detect squats in the ABA signals of the SMW. A logistic regression classifier [13] predicting 'squat' or 'no squat' from the spectrogram of the ABA signal containing $93 \times 29$ acceleration values in $\mathrm{dB}$ (spatial resolution $1 \mathrm{~mm}$, frequency resolution $22 \mathrm{~Hz}$, overlap 95\%) has been trained using in total 18,427 spectrograms of simulated and measured data. Applied on the measured ABA signals from the shunting yard Munich-Nord, the algorithm classifies spectrograms from all three severe squats correctly in all eight pass-bys. This corresponds to totally 28 squat passages since the signal from one axle box was corrupted and squat passages were discarded when the train velocity deviated more than $5 \%$ from the targeted $80 \mathrm{~km} / \mathrm{h}$. Concerning the four light squats, the spectrograms are classified correctly in $87 \%$ of the totally 31 squat passages. For the test of each squat, all data from this specific squat were removed from the training data. The algorithm also detects deteriorated insulated rail joints as 'squats'. Presumably, discrimination between squats and insulated rail joints would be possible when more data of insulated rail joints were available. Besides insulated rail joints, only a few (possibly) false alarms occurred. On two $250 \mathrm{~m}$ test sections of the measured signals, totally four 'squats' were detected at positions were no squats had been found in the visual inspection. Practically, these can all be discarded due to low peak accelerations below $40 \mathrm{~m} / \mathrm{s}^{2}$. 


\section{Conclusions}

In this paper, the acoustic signatures of squats in the ABA signals of the DB noise measurement car SMW have been determined from measurements and simulations with the model WERAN. Good agreement has been found between both. Based on these results, a logistic regression classifier to detect squats in the ABA signals of the SMW has been proposed. It achieves high hit rates for both severe and light squats. More data must, however, be collected to discriminate squats from deteriorated insulated rail joints.

\section{Acknowledgements}

This work has been carried out in the project AMONTRACK that has received funding from the European Union's Horizon 2020 research and innovation programme under the Marie Skłodowska-Curie grant agreement No. 744605.

\section{References}

1. Thompson, D.: Railway noise and vibration. Elsevier, Oxford, UK (2009).

2. Nielsen, J.C.O., Pieringer, A., Thompson, D.J., Torstensson, P.T.: Wheel-rail impact loads, noise and vibration: a review of excitation mechanisms, prediction methods and mitigation measures, In: Proceedings of IWRN13, Ghent, Belgium (2019).

3. Molodova, M., Li, Z., Núñez, A., Dollevoet, R.: Monitoring the railway infrastructure: Detection of surface defects using wavelets. In: Proc. of the 16th Int. IEEE Annual Conference on Intelligent Transportation Systems, The Hague, The Netherlands (2013).

4. An, B., Wang, P., Xu, J., Chen, R., Cui, D.: Observation and simulation of axle box acceleration in the presence of rail weld in high-speed monitoring. Appl. Sci. 7(12), 1259 (2017).

5. Schwanen, W., Kuijpers, A.H.W.M.: Early rail defect detection using sound measurements. In: J. Pombo (ed.) Proc. of the Third Int. Conference on Railway Technology: Research, Development and Maintenance, Civil-Comp Press, Stirlingshire, UK (2016).

6. Asmussen, B., Onnich, H., Strube, R., Greven, L.M., Schröder, S., Jäger, K., Degen, K.G: Status and perspectives of the "specially monitored track". J Sound Vib 293, 1070-1077 (2006).

7. Pieringer, A., Kropp W., Nielsen, J.C.O.: The influence of contact modelling on simulated wheel/rail interaction due to wheel flats. Wear 314(1-2), 273-281 (2014).

8. Pieringer, A: A numerical investigation of curve squeal in the case of constant wheel/rail friction. J. Sound Vib. 333(18), 4295-4313 (2014).

9. Kalker, J.J.: Three-Dimensional Elastic Bodies in Rolling Contact, Kluwer Academic Publishers, Dordrecht, Boston, London (1990).

10. Nielsen, J.C.O., Igeland, A.: Vertical dynamic interaction between train and track - influence of wheel and track imperfections, J. Sound Vib. 187(5), 825-839 (1995).

11. Thompson, D. et al.: Assessment of measurement-based methods for separating wheel and track contributions to railway rolling noise. Applied Acoustics 140, 48-62 (2018).

12. Andersson, R., Torstensson, P.T., Kabo, E., Larsson, F.: The influence of rail surface irregularities on contact forces and local stresses. Vehicle System Dynamics 53(1), 68-87 (2015).

13. Ng, A.: Machine Learning. Online course at Coursera Platform, Stanford University, https://www.coursera.org/learn/machine-learning, Accessed 20 May 2019. 